\title{
CALCULATION OF AGARD WING 445.6 FLUTTER USING NAVIER-STOKES AERODYNAMICS
}

\author{
Elizabeth M. Lee-Rausch \\ John T. Batina \\ NASA Langley Research Center \\ Hampton, Virginia 23681-0001
}

AIAA Paper No. 93-3476

Presented at the

AIAA 11th Applied Aerodynamics Conference

Monterey, California

August 9-11, 1993 



\title{
CALCULATION OF AGARD WING 445.6 FLUTTER USING NAVIER-STOKES AERODYNAMICS
}

\author{
Elizabeth M. Lee-Rausch* \\ John T. Batina** \\ NASA Langley Research Center \\ Hampton, Virginia 23681-0001
}

\begin{abstract}
The flutter characteristics of the first AGARD standard aeroelastic configuration for dynamic response, Wing 445.6, are studied using an unsteady Navier-Stokes algorithm in order to investigate a previously noted discrepancy between Euler flutter characteristics and the experimental data. The algorithm, which is a three-dimensional, implicit, upwind Euler/Navier-Stokes code (CFL3D Version 2.1), was previously modified for the time-marching, aeroelastic analysis of wings using the unsteady Euler equations. These modifications include the incorporation of a deforming mesh algorithm and the addition of the structural equations of motion for their simultaneous time integration with the governing flow equations. In this paper, the aeroelastic method is extended and evaluated for applications that use the NavierStokes aerodynamics. The paper presents a brief description of the aeroelastic method and presents unsteady calculations which verify this method for Navier-Stokes calculations. A linear stability analysis and a time-marching aeroelastic analysis are used to determine the flutter characteristics of the isolated $45^{\circ}$ swept-back wing. Effects of fluid viscosity, structural damping, and number of modes in the structural model are investigated. For the linear stability analysis, the unsteady generalized aerodynamic forces of the wing are computed for a range of reduced frequencies using the pulse transfer-function approach. The flutter characteristics of the wing are determined using these unsteady generalized aerodynamic forces in a traditional V-g analysis. This stability analysis is used to determine the flutter characteristics of the wing at free-stream Mach numbers of 0.96 and 1.141 using the generalized aerodynamic forces generated by solving the Euler equations and the Navier-Stokes equations. Time-marching aeroelastic calculations are performed at a free-stream Mach number of 1.141 using the Euler and Navier-Stokes equations to compare with the linear V-g flutter analysis method. The V-g analysis, which is used in conjunction with the time-marching analysis, indicates that the fluid viscosity has a significant effect on the

\footnotetext{
*Research Engineer, Aeroelastic Analysis and Optimization Branch, Structural Dynamics Division. Member AIAA.

${ }^{* * *}$ Senior Research Scientist, Aeroelastic Analysis and Optimization Branch, Structural Dynamics Division. Associate Fellow AIAA.

Copyright (C) 1993 by the American Institute of Aeronautics and Astronautics, Inc. No copyright is asserted in the United States under Title 17, U.S. Code. The U.S. Government has a royalty-free license to exercise all rights under the copyright claimed herein for Governmental purposes. All other rights are reserved by the copyright
} owner.
\end{abstract}

supersonic flutter boundary for this wing while the structural damping and number of modes in the structural model have a lesser effect.

\section{$\underline{\text { Nomenclature }}$}

\begin{tabular}{|c|c|}
\hline$A_{i j}$ & $\begin{array}{l}\text { generalized aerodynamic force resulting from } \\
\text { pressure induced by mode } \mathrm{j} \text { acting through } \\
\text { mode } \mathrm{i}\end{array}$ \\
\hline$b$ & root semichord \\
\hline$c$ & root chordlength \\
\hline$C_{p}$ & pressure coefficient \\
\hline$g_{i}$ & structural damping for mode i \\
\hline$k$ & reduced frequency, $\frac{\omega c}{2 U_{\infty}}$ \\
\hline$M_{\infty}$ & free-stream Mach number \\
\hline$Q$ & free-stream dynamic pressure \\
\hline$R e_{c}$ & $\begin{array}{l}\text { free-stream Reynolds number based on root } \\
\text { chordlength }\end{array}$ \\
\hline$T$ & dimensional time \\
\hline$U_{F}$ & flutter speed \\
\hline$U_{\infty}$ & streamwise free-stream speed \\
\hline$\alpha$ & angle of attack \\
\hline$\eta$ & nondimensional semispan location \\
\hline$\mu$ & mass ratio \\
\hline$\omega$ & angular frequency \\
\hline$\omega_{\alpha}$ & $\begin{array}{l}\text { uncoupled natural frequency of the wing first } \\
\text { torsion mode }\end{array}$ \\
\hline
\end{tabular}

\section{Introduction}

From the first calculations of Ballhaus and Goorjian ${ }^{1}$ to more recent applications of the Euler and Navier-Stokes equations for the aeroelastic analysis of three-dimensional wings, the field of computational aeroelasticity has progressed rapidly. ${ }^{2}$ With the advent of more powerful computers and more efficient algorithms, researchers have in recent years computed more detailed and sophisticated simulations of aeroelastic phenomena. Because of their relative computational efficiency, the transonic small distur- 
bance (TSD) equation and the full potential (FP) equation have been applied to a wider variety of three-dimensional configurations than the Euler and Naiver-Stokes equations. ${ }^{2}$ Similarly, the TSD and FP equations also have been utilized for more detailed analyses of the aeroelastic characteristics of these configurations. In contrast, the higher-order methods, such as the Euler and Navier-Stokes equations, typically have been applied to the analyses of flexible wings over a limited range of flow conditions. ${ }^{3-11}$ Only recently, the Euler equations have been used to compute the flutter boundary for the AGARD standard aeroelastic configuration for dynamic response, Wing 445.6. ${ }^{12,}{ }^{13}$ In Ref. 12, an unstructured-grid Euler code is used to compute the flutter characteristics of Wing 445.6 for free-stream Mach numbers ranging from 0.499 to 0.96 , and the computed flutter speeds and frequencies are compared with the experimental values measured for this wing. In Ref. 13, Lee-Rausch and Batina compute the complete flutter boundary for the same wing using the Euler equations on a structured grid. Calculated flutter results are compared with experimental data for seven free-stream Mach numbers, which define the flutter boundary over a range of Mach number from 0.499 to 1.14 (See Fig. 1.) These comparisons show good agreement in flutter characteristics for free-stream Mach numbers below one. However, for free-stream Mach numbers above one, the computed aeroelastic results predict a premature rise in the flutter boundary as compared with the experimental boundary. The purpose of this paper is to extend the capability presented in Ref. 13 and to compute the flutter characteristics of the AGARD Wing 445.6 using the Navier-Stokes equations so that the source of the discrepancy in the flutter characteristics at the supersonic free-stream Mach numbers can be investigated.

Many of the aeroelastic analyses performed with the nonlinear flow equations have been obtained by calculating the transient response of the coupled aerodynamic/structural system. Other analyses have been performed using harmonic loads. ${ }^{14-17}$ In the harmonic analysis, the unsteady aerodynamic forces are assumed to be locally linear and a traditional stability analysis is used to determine flutter characteristics based on the calculated harmonic loads. One advantage of the linear stability analysis is that it offers a significant computational savings over the time-marching analysis if the number of modes necessary to model the structure is small and if there are no static aeroelastic deformations. Another advantage of the linear stability analysis is that it can provide more information regarding the effects of individual modes and the effects of structural damping from fewer computations than the time-marching approach. It is important to note, however, that the assumption of superposition of airloads may not be accurate in some cases such as those involving large amplitudes of motion and separated flow. However, in previous studies, ${ }^{14,15,17}$ for cases with low angles of attack and small amplitudes of motion, the flutter characteristics obtained from time-marching analyses com-
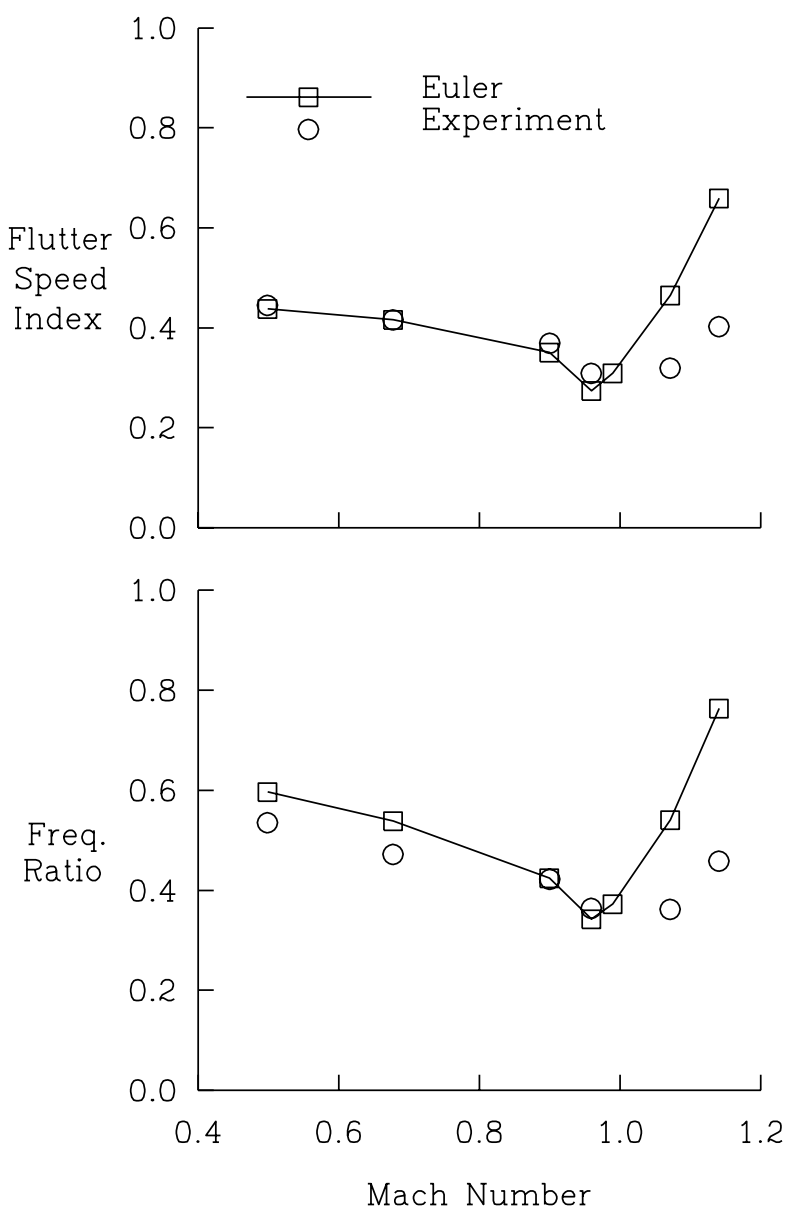

Figure 1 Comparison of computed Euler flutter results from Ref. 13 with experimental data for Wing 445.6.

pare favorably with those obtained from a harmonic analysis. For these reasons, the linear stability analysis is used in conjunction with time-marching analyses to study the aeroelastic characteristics of the AGARD Wing 445.6 using the Navier-Stokes equations.

In Ref. 13, modifications are made to an existing threedimensional, unsteady Euler/Navier-Stokes code (CFL3D Version 2.1) for the aeroelastic analysis of wings. These modifications include the incorporation of a deforming mesh algorithm and the addition of the structural equations of motion for their simultaneous time integration with the governing flow equations. This paper gives a brief description of these modifications and presents unsteady calculations which verify the modifications to the code for the solution of the Navier-Stokes equations. Results from calculations performed about a rigid wing undergoing forced plunging and pitching motions are presented to verify the performance of the deforming mesh algorithm. A linear stability analysis and a time-marching aeroelastic analysis are used to determine the flutter characteristics of the wing. Effects of fluid viscosity, structural damping, and number of modes in the structural model are investigated. For the linear stability analysis, the unsteady generalized aerodynamic forces 
(GAF's) of the wing are computed for a range of reduced frequency using a pulse transfer-function analysis. The flutter characteristics of the wing are determined using these unsteady GAF's in a traditional V-g analysis. This stability analysis is used to determine the flutter characteristics of the wing at free-stream Mach numbers of 0.96 and 1.141 using the GAF's generated with the Euler equations and with the Navier-Stokes equations. In addition, time-marching aeroelastic calculations are performed at a free-stream Mach number of 1.141 using the Euler and Navier-Stokes equations for comparison with the linear V-g flutter analysis results. The time-marching Euler flutter characteristics are recomputed on a mesh that is similar to the Navier-Stokes mesh in order to more effectively isolate the effects of viscosity. Computed flutter results are compared with the experimental data and with other computational results from Ref. 13 to demonstrate the aeroelastic capability for the Navier-Stokes equations and to evaluate the effects of viscosity on the flutter characteristics at these flow conditions.

\section{Time-Marching Aeroelastic Analysis}

The time-marching aeroelastic procedure used in this study is typical of those currently in use. ${ }^{11,12,18}$ In general, the aeroelastic equations of motion are formulated in terms of a finite modal series of free-vibration modes. These equations then are written in terms of a linear state-space equation such that a modified state-transition-matrix integrator can be used to march the coupled fluid-structural system forward in time. The fluid forces are coupled with the structural equations of motion through the generalized aerodynamic forces. To determine the flutter conditions at a given free-stream Mach number, aeroelastic transients are computed at several values of dynamic pressure which bracket the flutter point. The frequency and damping characteristics of the transient responses at each dynamic pressure are determined from a least squares curve fit, ${ }^{19}$ and the flutter dynamic pressure and frequency associated with this Mach number can be estimated by interpolation.

The time-marching aeroelastic procedure for a problem utilizing the Navier-Stokes equations is almost identical to the procedure described in more detail in Ref. 13 for the Euler equations. However, when using the Navier-Stokes equations, a free-stream Reynolds number must be specified for the static and dynamic computations at each dynamic pressure of interest. This requirement raises the issue of how to vary the dynamic pressure in a computational fluid dynamics, time-marching aeroelastic analysis to obtain the flutter conditions for a specific free-stream Mach number. When comparing with experimental data, either the free-stream density or the free-stream velocity can be deviated from the experimental value to obtain variation in the dynamic pressure. Changing the density or velocity will, however, change the Reynolds number associated with that flow condition. An analysis of each of these options for

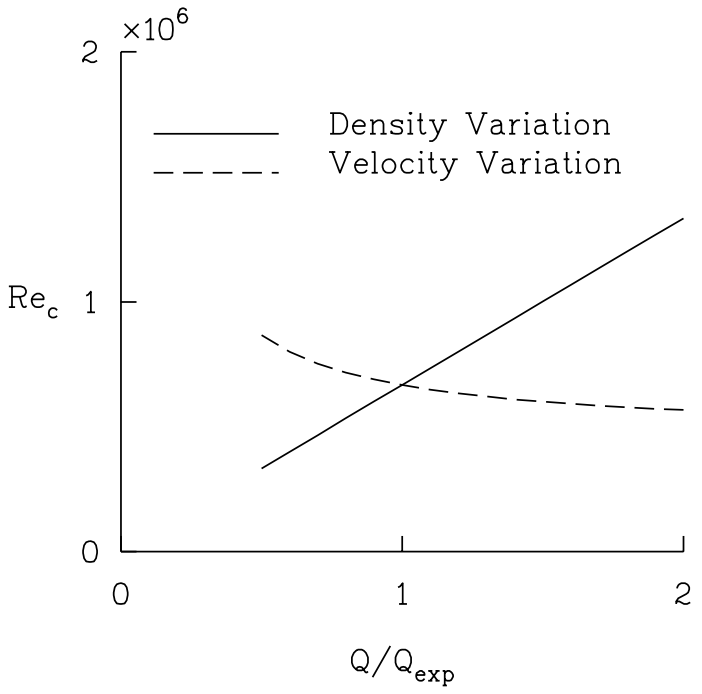

(a) $M_{\infty}=0.96$.



(b) $M_{\infty}=1.141$.

Figure 2 Variation in free-stream Reynolds number with variation in dynamic pressure for time-marching aeroelastic calculations.

the experimental flutter conditions of the Wing 445.6 at $M_{\infty}=0.96$ and 1.141 is shown in Figs. 2(a) and (b), respectively. Figures 2(a) and (b) show the variation in the free-stream Reynolds number $\left(R e_{c}\right)$ with the variation in the free-stream dynamic pressure nondimensionalized by the experimental flutter dynamic pressure $\left(Q / Q_{e x p}\right)$. Figures 2(a) and (b) indicate that for a constant free-stream density, varying the free-stream velocity results in a smaller variation in Reynolds number than maintaining a constant velocity while varying the density (especially for conditions above the experimental dynamic pressures). Varying the free-stream velocity for a given Mach number does, however, require a 
change in the free-stream temperature and, subsequently, the adiabatic wall temperature. The effect of Reynolds number variation on the time-marching cases considered in this study is discussed later in the paper.

\section{Linear Stability Analysis}

A conventional V-g method is used in this study for computing flutter speeds and frequencies. This method interpolates the given generalized aerodynamic forces (GAF's) to compute the eigenvalues of the flutter determinant. The procedure used to obtain the flutter eigenvalues is contained in the parametric flutter analysis program FLUTDET which is part of the FAST flutter analysis package. ${ }^{20}$ The capabilities and techniques used in FLUTDET are described in Ref. 21. Although FLUTDET originally was designed to interface with GENFLU, which calculates unsteady aerodynamic forces from subsonic kernel matrices, compatible input from other aerodynamic methods can be used. In addition to the GAF's, the generalized mass and modal stiffnesses are required input to FLUTDET. The program generates flutter characteristics and $\mathrm{V}-\mathrm{g}$ plots for each specified free-stream density.

Traditional flutter analysis methods, such as the V-g method, assume that the unsteady aerodynamic forces are locally linear and utilize modal superposition of harmonic loads. Linear aerodynamic methods typically compute the GAF's as functions of reduced frequency. For nonlinear aerodynamic methods, these GAF's are obtained from timemarching calculations by computing several cycles of a forced harmonic oscillation and using the last cycle of oscillation to determine the first harmonic component of the GAF's. Multiple time-marching computations are required at various reduced frequencies to generate the GAF's for each structural mode used in the flutter analysis.

An alternate method for determining the GAF's is the pulse transfer-function analysis. In contrast to the forced harmonic method, the pulse analysis can determine the GAF's over a range of reduced frequency in a single timemarching calculation for each mode. In the pulse analysis, the unsteady forces are determined from the response due to motion represented by a smoothly varying, exponentially shaped pulse. A fast Fourier transform of the unsteady force is divided by the fast Fourier transform of the displacement to obtain the GAF. Transonic small disturbance results computed using the pulse analysis for a pitching flat plate are found to be in good agreement with linear theory calculations. $^{22}$ Also in Ref. 22, the GAF's of airfoils at transonic speeds computed from a pulse analysis are shown to be in good agreement with the GAF's computed using the harmonic method which tends to verify that the analysis is valid for predicting the linear small perturbation response about a nonlinear flowfield. Similarly in Refs. 11 and 23, Euler results for a pitching and plunging airfoil show good agreement between the pulse and harmonic methods. Therefore, because of its computational efficiency, the pulse transferfunction method is used in this study to compute the GAF's for input to the V-g analysis.

For linear methods, the GAF's for a vibration mode are functions of free-stream Mach number, planform (geometry), and reduced frequency. For nonlinear methods such as the TSD and Euler equations, if the assumptions of local linearity are maintained, then this functionality also will hold true. However, for the Navier-Stokes equations an additional parameter must be considered: the free-stream Reynolds number. The V-g stability analysis does not ensure that the Reynolds number associated with the computation of the GAF's will match the Reynolds number associated with the computed flutter condition. The only way to ensure a matched Reynolds number solution is to iterate between the calculations of the GAF's and the V-g analysis. This option is not feasible for a Navier-Stokes analysis due to constraints on computational resources. However, for the cases analyzed in this study, it was not considered to be a necessary requirement for the following reason. In the V-g analysis, the flutter speed is computed for a given free-stream density. Typically when comparing with experimental data, the experimental free-stream density is specified. As discussed in the previous section, variations in free-stream Reynolds number due to changes in free-stream velocity are small. If the GAF's are computed at the experimental Reynolds number, the difference between the experimental Reynolds number and the Reynolds number based on the computed flutter characteristics are small for the cases considered in this study. These differences are discussed later in the paper.

\section{Upwind Euler/Navier-Stokes Algorithm}

The CFL3D ${ }^{24-26}$ code uses a three-factor, implicit, finite-volume algorithm based on upwind-biased spatial differencing to solve the time-dependent Euler equations and thin-layer Navier-Stokes equations. The algorithm, which is a cell-centered scheme, uses upwind differencing based on either flux-vector splitting or flux-difference splitting. Both types of upwind differencing account for the local wavepropagation characteristics of the flow and sharply capture shock waves. Also, because these schemes are naturally dissipative, additional artificial dissipation terms are not necessary. Several types of flux limiting are available within the code to prevent oscillations in the solution near shock waves which are typically found in higher-order schemes. For applications utilizing the thin-layer Navier-Stokes equations, two turbulence models are available: the equilibrium, algebraic, eddy-viscosity model of Balwin-Lomax, ${ }^{27}$ and the nonequilibrium half-equation model of Johnson and King. ${ }^{28}$ For unsteady cases, the original algorithm contains the necessary metric terms for a rigidly translating and rotating mesh which moves without deforming. For cases involving a deforming mesh, however, an additional term accounting for the change in cell volume must be included in the time- 
discretization of the governing equations. This modification is implemented as described in Ref. 11.

\section{Deforming Mesh Algorithm}

In the time-marching aeroelastic calculations and in the modal pulse transfer-function calculations, the mesh must be updated at every time level so that it conforms to the aeroelastically deformed shape of the wing. Because the aeroelastic motions of the wing are arbitrary in nature, a general mesh updating procedure is necessary. One such method, the deforming mesh algorithm, models the mesh as a network of springs and solves the static equilibrium equations for this network to determine the new locations of the mesh grid points. This algorithm was originally developed by Batina $^{29}$ for tetrahedral cells and extended by Robinson et al. ${ }^{11}$ for hexahedral cells. The method described in Ref. 11 was used by the current authors for time-marching aeroelastic calculations on Euler grids. ${ }^{13}$ This algorithm is extended in the current study for use on Navier-Stokes meshes. The basic principles of the method do not change although some modifications to the deforming mesh boundary conditions are required as described below.

For the deforming mesh algorithm, the edge of each hexahedral cell is modeled as a spring with a stiffness that is inversely proportional to a power of the length of the edge. Diagonal springs are added along the faces of each cell in order to prevent cell shearing. Similarly, the stiffness of these springs is inversely proportional to a power of the length of the diagonal. As suggested in Ref. 11, a power of three was used in the present calculations. At each time level, the grid points on the outer boundary are held fixed, and the displacement of the wing surface is specified. For aeroelastic calculations, the displacement is determined from the integration of the structural equations of motions. The new locations of the interior grid points then are determined by solving the static equilibrium equations which result from a summation of forces at each grid point in the $\mathrm{x}, \mathrm{y}$ and $\mathrm{z}$ coordinate directions. These static equilibrium equations are solved using a predictor-corrector method. The new grid point locations are first predicted by an extrapolation from the previous two time levels and then corrected using several Jacobi iterations of the static equilibrium equations. In previous Euler calculations, four Jacobi iterations were sufficient to move the mesh. ${ }^{11,13}$ For the current Euler and Navier-Stokes calculations, additional Jacobi iterations were required to move the mesh. The Euler calculations required up to 8 iterations, and the Navier-Stokes calculations required up to 12 iterations.

Because the dynamic mesh is modeled using structural equations, this model must represent a realistic structure. There is one case where this has been found to be a problem. In a C-H-type mesh for an isolated wing, chordwise C-type meshes are stacked along the span to form the threedimensional mesh. As these C-type meshes transition from the wing surface to the exterior flow field the airfoil section is collapsed to zero thickness to form a "tip wake" surface. The points around the "leading-edge" of this tip wake surface must negotiate a $360^{\circ}$ turn. The structural equations modeling the mesh in this area lack stiffness in one direction. This, in turn, causes poor convergence rates for the predictor-corrector procedure. It was found that specifying the location of the leading-edge points for the tip wake alleviates this problem. When using a $\mathrm{C}$-H-type mesh, another problem occurs for Navier-Stokes applications. In this case, the flow solution is very sensitive to the angle of intersection between the trailing edge of the airfoil and the trailing-edgewake line of points. It was found that, to obtain accurate unsteady solutions, the slope of the trailing-edge wake must match the slope of the instantaneous airfoil camber line at the trailing edge. These slopes were matched by specifying the points in the wake using a quadratic function.

\section{AGARD Wing 445.6}

The first AGARD standard aeroelastic configuration for dynamic response, Wing $445.6,{ }^{30}$ was tested in the $16-$ foot Transonic Dynamics Tunnel (TDT) at the NASA Langley Research Center. ${ }^{31}$ The wing had a quarter-chord sweep angle of $45^{\circ}$, a panel aspect ratio of 1.65 , a taper ratio of 0.66 , and a NACA 65A004 airfoil section. Several models of the wing were tested in the TDT including full span and semi-span models. The model used in this study is a semi-span, wall-mounted model which was constructed of laminated mahogany. The root chord of this model was 1.833 feet and the semi-span was 2.5 feet. In order to obtain flutter data for a wide range of Mach number and density conditions in the TDT, holes were drilled through the mahogany wing to reduce its stiffness. The aerodynamic shape of the original wing was preserved by filling these holes with rigid foam plastic. Flutter data for this model tested in air are reported in Ref. 31 over a range of Mach number from 0.338 to 1.141 . Natural boundary-layer transition was allowed throughout the test. The semi-span model was attached directly to the wind tunnel wall (no splitter plate was used); therefore, the wing root was immersed in the wall boundary layer. Ref. 30 indicates that the displacement thickness of the wall boundary layer was 0.8 inch or less.

As in Ref. 13, the first four natural vibration modes of the wing are used to model the wing structure. These modes, numbered 1 through 4 , represent first bending, first torsion, second bending, and second torsion, respectively, as determined by a finite element analysis. ${ }^{30}$ The modes have natural frequencies of $9.6 \mathrm{~Hz}$ for the first bending mode, $38.17 \mathrm{~Hz}$ for the first torsion mode, $48.35 \mathrm{~Hz}$ for the second bending mode, and $91.54 \mathrm{~Hz}$ for the second torsion mode as determined from a ground vibration test. Because the maximum amplitudes of motion were small for these tests, the motion due to the first four vibration modes was dominated by out-of-plane (or vertical) displacements. Therefore in the 


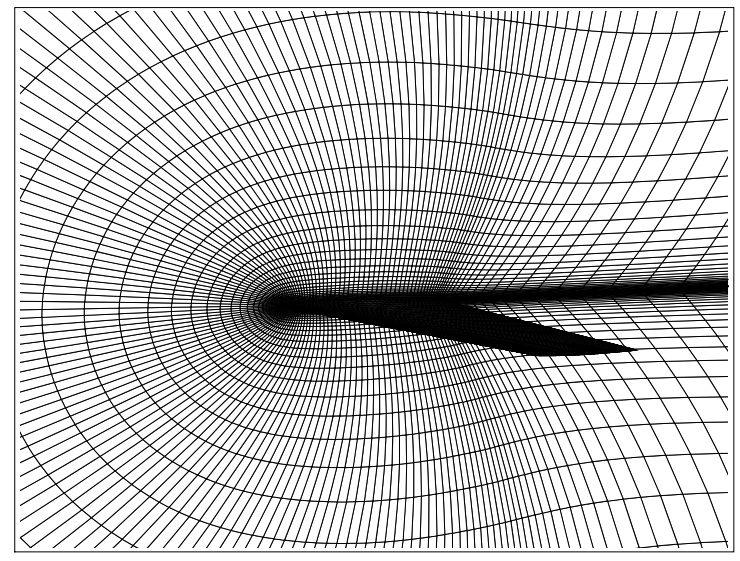

(a) Euler.

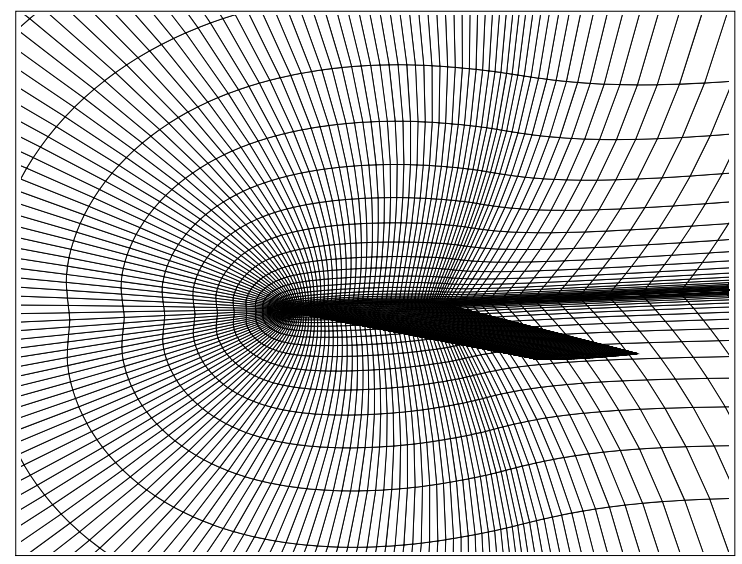

(b) Navier-Stokes.

Figure 3 Partial view of the $193 \times 41 \times 65$ computational grids on the wing surface and symmetry plane.

computational analysis, the modal deflections are defined only by their out-of-plane deflections.

\section{Results and Discussion}

Steady and unsteady Euler and Navier-Stokes results are computed at free-stream Mach numbers $M_{\infty}=0.96$ and 1.141. The steady-state viscous calculations are performed at the experimental flutter conditions which corresponded to a free-stream Reynolds number of 364,600 per foot for the $M_{\infty}=0.96$ calculation and a free-stream Reynolds number of 537,700 per foot for the $M_{\infty}=1.141$ calculation. As mentioned in a previous section, the GAF's at each free-stream Mach number also are computed using the experimental flutter conditions. Two cases of viscous timemarching flutter calculations at $M_{\infty}=1.141$ are considered. In one case, the Reynolds number is specified as the experimental value for calculations at each dynamic pressure. In the other case, the Reynolds number is varied with the dynamic pressure.

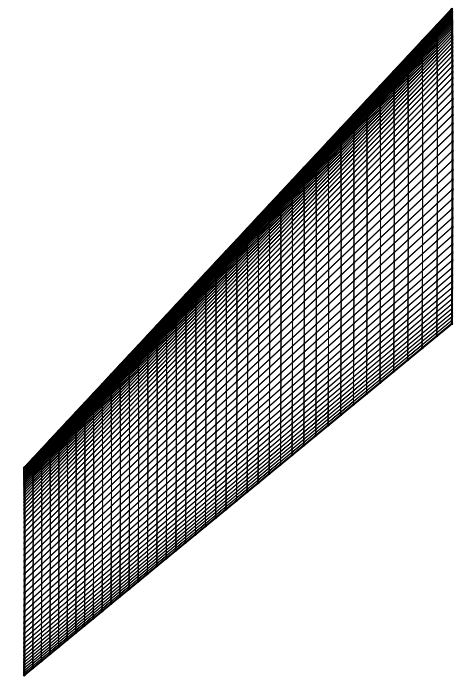

Figure 4 Planform view of computational grids.

The Euler and Navier-Stokes computations are performed using a 193 x 41 x 65 C-H-type grid with 193 points wrapped around the wing and its wake (129 points on the wing surface), 65 points distributed from the wing root to the spanwise boundary (41 points on the wing surface), and 41 points distributed radially from the wing surface to the outer boundary. This mesh topology is chosen rather than the O-O-type topology because the wind tunnel model has a sheared-off tip. Both grids extend 6 root chord lengths from the wing to the upstream boundary, 7 root chord lengths from the wing to the downstream boundary, 6 root chord lengths from the wing to the upper and lower boundaries, and 1 semi-span length from the tip to the side boundary. A partial view of the surface mesh on the wing and symmetry plane for the Euler and the Navier-Stokes grids is shown in Figs. 3(a) and (b), respectively. The Euler and Navier-Stokes grids have identical chordwise and spanwise distributions. Therefore, the surface mesh for both grids is the same. A planform view of the surface mesh for both the Euler grid and the Navier-Stokes grid is shown in Fig. 4. The distribution of points in the radial direction is not the same for the two grids. For the Euler grid, the grid spacing normal to the surface is 0.1 percent of the local chord. For the Navier-Stokes grid, the grid spacing normal to the surface is varied over the chord such that at least one point will be in the laminar sublayer of the turbulent boundary layer. An additional finer Navier-Stokes mesh is utilized to compute steady-state results in a grid density study. This mesh is a $265 \times 81 \times 65 \mathrm{C}$-H-type mesh identical in topology to the $193 \times 41 \times 65$ mesh. The fine mesh has 177 points wrapped around the wing chord and 41 points distributed from the wing root to tip.

For all of the calculations, the Euler and Navier-Stokes equations are solved using flux-vector splitting and a smooth flux-limiter. Convergence to steady-state is accelerated using local time-stepping, mesh sequencing and multi-grid cycling. For time-marching calculations, the nondimensional 


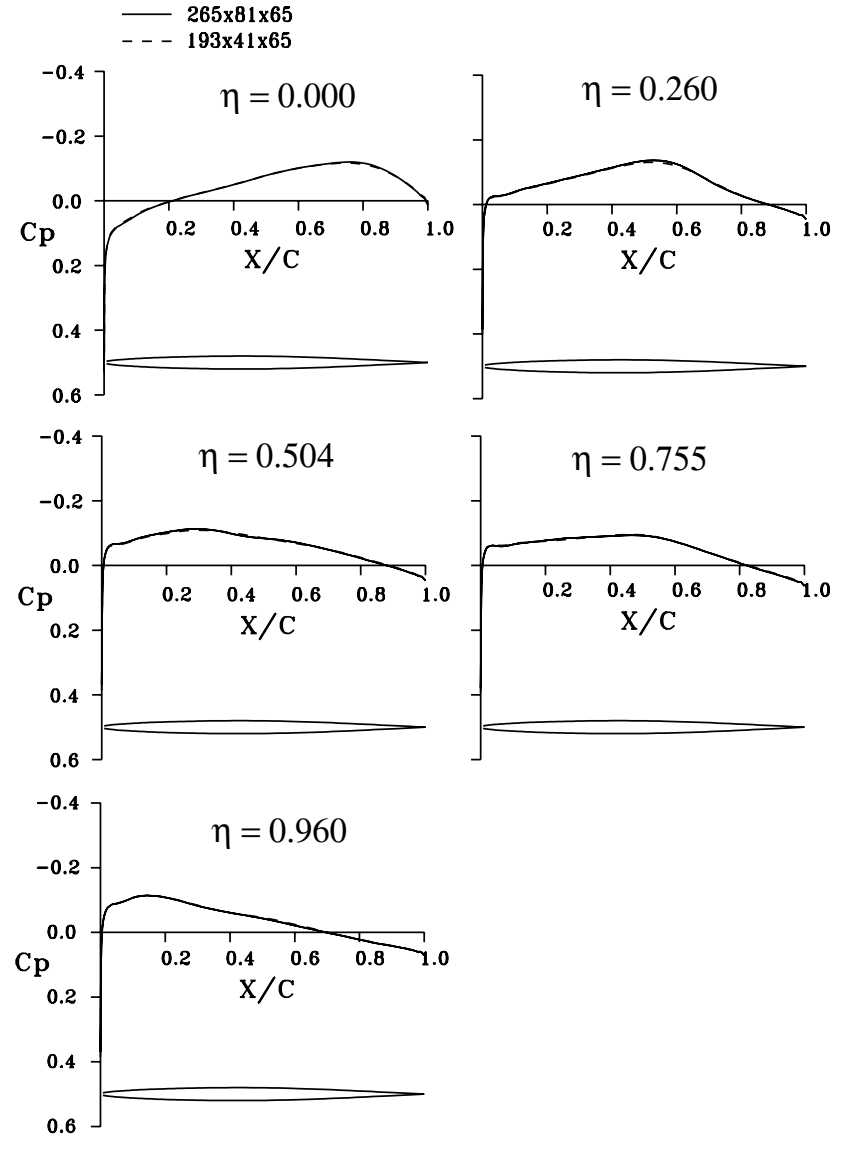

(a) $M_{\infty}=0.96$.

Figure 5 Comparison of steady-state pressure coefficient distributions between the $265 \times 81 \times 65$

C-H mesh and the 193 x 41 × 65 C-H mesh.

global time step (based on the root chord and the free-stream speed of sound) is 0.03273 for both the Euler and the NavierStokes calculations. Viscous calculations are performed using the Baldwin-Lomax turbulence model with an adiabatic wall temperature. Since no information is available on the experimental transition location, turbulence is modeled over the entire wing surface. This is a good approximation for transition locations near the leading edge of the wing.

\section{Steady-State Results}

Before beginning the flutter analyses of the wing, a grid density study was performed for the steady-state viscous flow fields. Results from the $265 \times 81 \times 65 \mathrm{C}-\mathrm{H}$ mesh with approximately 1.4 million grid points were used as a bench mark. After some investigation with different grid densities, the $193 \times 41 \times 65 \mathrm{C}-\mathrm{H}$ mesh with 0.51 million points was found to predict very similar steady-state surface pressure distributions as the finer mesh for $M_{\infty}=0.96$ and for $M_{\infty}=1.141$. Figures 5(a) and (b) show the surface pressure coefficients at five span stations corresponding to the 0.0 percent, 26.0 percent, 50.4 percent, 75.5 percent,

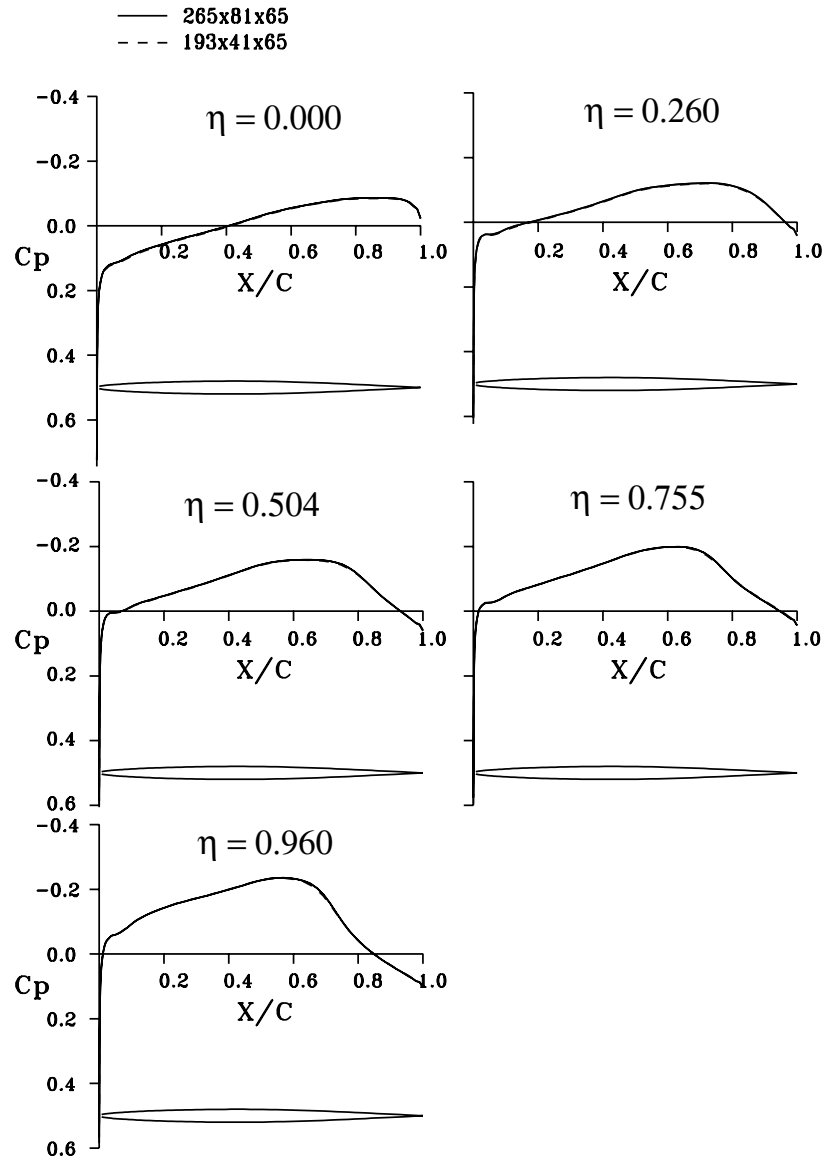

(b) $M_{\infty}=1.141$.

Figure 5 Concluded.

and 96.0 percent of the semispan for both grids. The surface pressure plots indicate that the coarser grid resolves the flow features across the span of the wing at least as well as the finer grid. For this reason, the $193 \times 41$ x 65 grid was chosen for this study, and all of the subsequent results presented in this paper were computed using this grid to minimize the cost of the aeroelastic computations.

Steady-state flowfields are used as initial conditions for the unsteady calculations. Comparisons of steady-state pressure coefficient contours of these initial flowfields on the upper wing surface are shown in Figs. 6 and 7 to illustrate the basic flow characteristics of the time-marching calculations at $M_{\infty}=0.96$ and 1.141 and $\alpha=0^{\circ}$. Regions of supercritical flow (indicated by the shaded areas) are determined in Figs. 6(a) and (b) by the critical pressure coefficient contour $\left(C_{p}^{*}=-0.0697\right)$. Although $C_{p}^{*}$ is derived from isentropic relations, it is used in this case to give an approximate indication of supersonic flow over the wing surface. For the Navier-Stokes calculation, $C_{p}^{*}$ is an indication of supersonic flow at the edge of the boundary layer based on the assumption that for an attached flow the gradient of pressure through the boundary layer is zero. The pressure coefficient contours, shown in Figs. 6(a) and (b), indicate that at $M_{\infty}=0.96$ a 


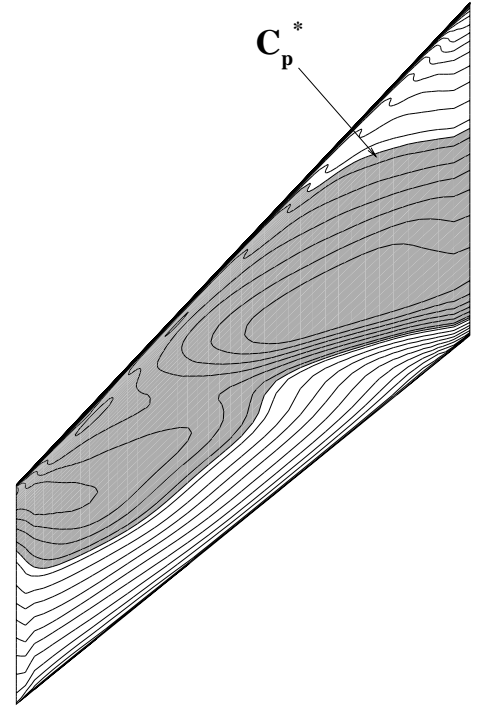

(a) Euler.

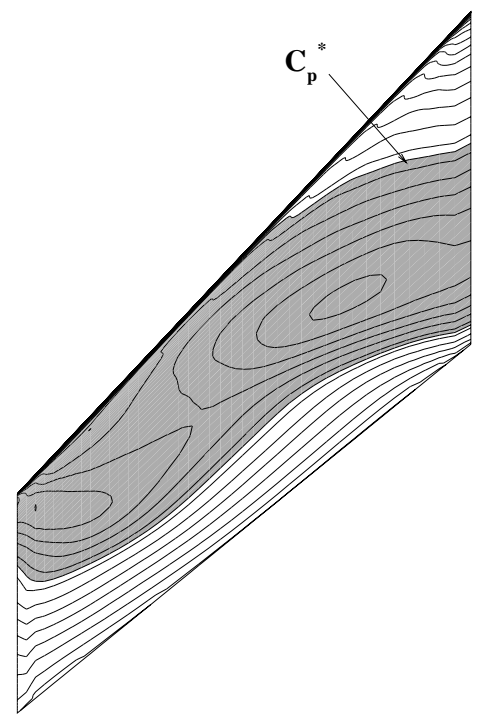

(b) Navier-Stokes.

Figure 6 Comparison of steady-state pressure coefficient contours on the upper surface of Wing 445.6 at $M_{\infty}=0.96$ and $\alpha=0^{\circ}$.

large area of supercritical flow has formed on the forward portion of the wing. This area of supercritical flow, however, does not terminate with a shock. Although, the Euler calculation predicts higher levels of acceleration over the wing (lower minimum pressure coefficient) and a more rapid recompression on the inboard portion of the wing, the Euler and Navier-Stokes calculations predict similar regions of supercritical flow.

Regions of supercritical flow are determined for $M_{\infty}=1.141$ in Figs. 7 (a) and (b) by the critical pressure



(a) Euler.

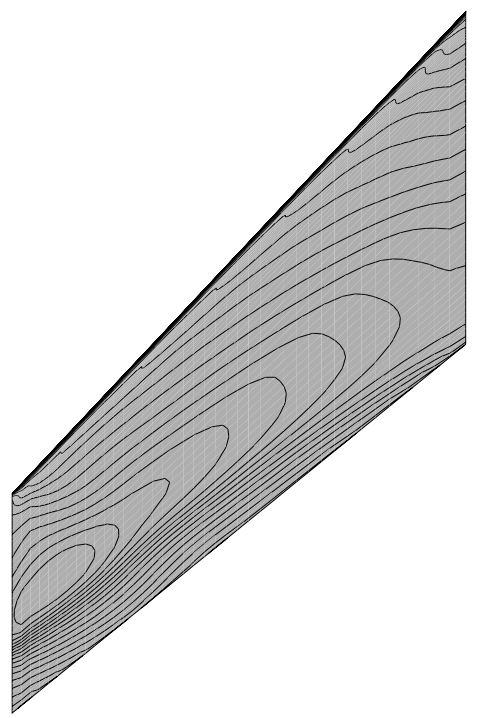

(b) Navier-Stokes.

Figure 7 Comparison of steady-state pressure coefficient contours on the upper surface of Wing 445.6 at $M_{\infty}=1.141$ and $\alpha=0^{\circ}$.

coefficient contour $\left(C_{p}^{*}=0.2057\right)$. At $M_{\infty}=1.141$, the supercritical region encompasses the entire wing surface except for a small area around the leading edge of the wing. Pressure coefficient contours shown in Fig. 7(a) indicate that for the inviscid computation, an oblique shock has formed on the aft portion of the wing. On the outboard portion of the wing, the shock is located at approximately 75 percent of the local chord. Pressure coefficient contours shown in Fig. 7(b) indicate that for the viscous computation, a weaker oblique shock forms on the outboard portion of the wing at approximately 70 percent of the local chord. A comparison 

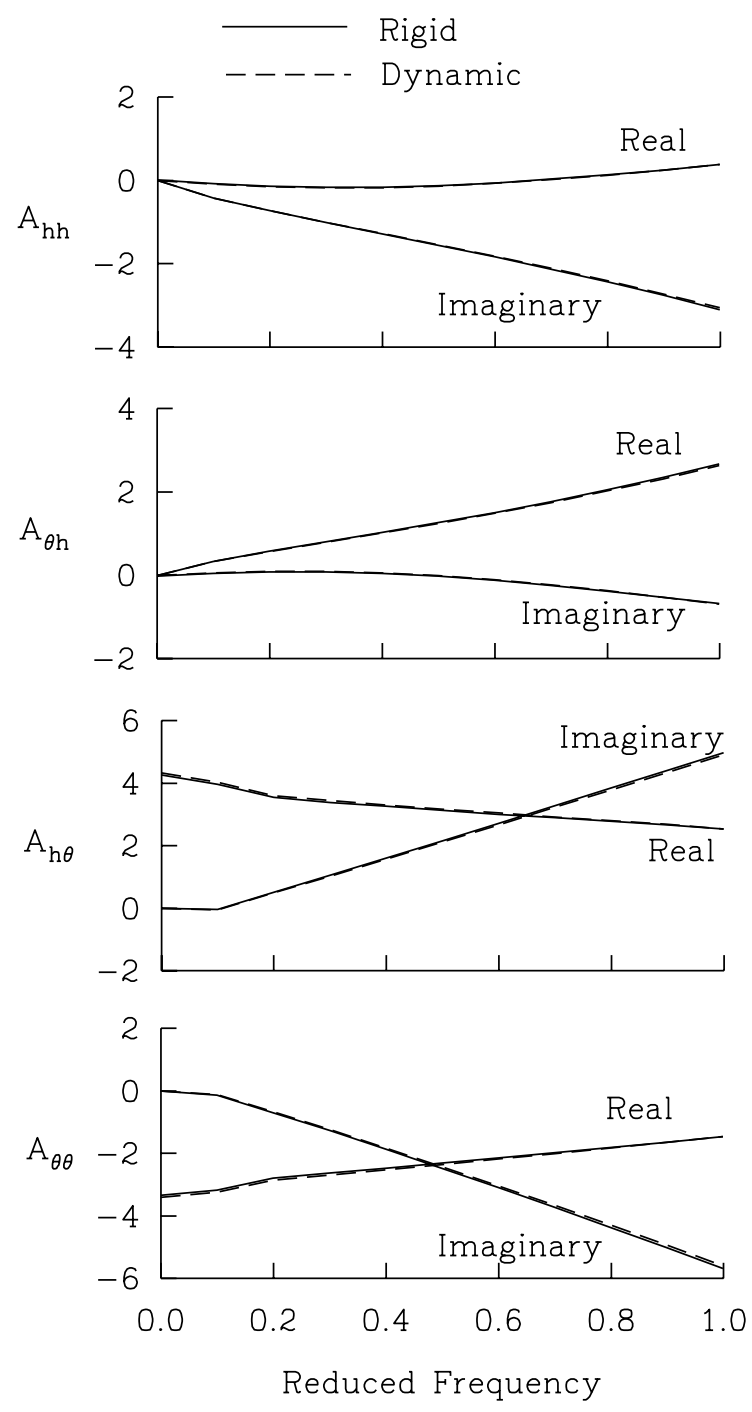

Figure 8 Comparison of generalized aerodynamic forces for the rigid plunge and pitch of Wing 445.6 at $M_{\infty}=0.96$ and $\alpha=0^{\circ}$ computed using the Navier-Stokes equations.

of Figs. 6 and 7 indicates that viscosity has a greater effect on the surface pressures at $M_{\infty}=1.141$ than at $M_{\infty}=0.96$. Significant changes in the steady-state flow conditions from $M_{\infty}=0.96$ to 1.141 are also indicated by the formation of a shock at the tip of the wing. This range of free-stream Mach number corresponds to the range of Mach numbers where the experimental flutter boundary and the computed flutter boundary in Ref. 13 rapidly rise (See Fig. 1.)

\section{Pulse Transfer-Function Results}

Rigid pitching and plunging The generalized forces for the wing at $M_{\infty}=0.96$ are computed for the viscous case using the pulse transfer-function analysis method described in a preceding section. The pulse calculations are restarted from a steady-state flow condition at $\alpha=0^{\circ}$. A plunging motion and a pitching motion about the root quarter-chord, which are defined as modes $h$ and $\theta$ respectively, are analyzed. These simple modes were chosen so that the motion of the wing could be simulated not only by the deforming mesh algorithm but also by a rigid translation and rotation of the grid. The maximum pitch amplitude is $1^{\circ}$, the maximum plunge amplitude is 0.01 root chord lengths, and the frequency resolution of the pulse analysis is 0.1 . The results of the pulse analysis for the plunging and pitching motions, shown in Fig. 8, are plotted as real and imaginary components of the unsteady forces, $A_{i j}$, as a function of the reduced frequency $k$ based on wing root semichord. These generalized forces represent work divided by the dynamic pressure and $c^{2}$. The generalized force $A_{h h}$ is the lift due to plunge, $A_{h \theta}$ is the lift coefficient due to pitching, $A_{\theta h}$ is the pitching moment due to plunge, and $A_{\theta \theta}$ is the pitching moment due to pitch. As shown in Fig. 8, the forces are independent of the way in which the mesh was moved which verifies the implementation and performance of the dynamic mesh algorithm for the Navier-Stokes computations. A similar analysis is performed in Ref. 13 to verify the implementation and performance of the dynamic mesh algorithm for Euler computations.

Structural modes The generalized forces for the wing at $M_{\infty}=0.96$ and 1.141 are computed for the inviscid and viscous cases using the pulse transfer-function analysis. The pulse calculations are restarted from a steady-state flow condition at $\alpha=0^{\circ}$. The first four structural modes are analyzed. The maximum amplitude of the generalized displacement is 0.02 for mode $1,0.005$ for mode 2, 0.02 for mode 3 , and 0.005 for mode 4 . These displacements result in a maximum deflection of approximately 0.50 inch at the wing tip. The results of the pulse analyses, shown in Figs. 9 and 10 , are plotted for the real and imaginary components of the unsteady forces $A_{i j}$ as functions of the reduced frequency. The frequency resolution of the pulse analyses is 0.04 .

A comparison of inviscid and viscous results in Fig. 9 for $M_{\infty}=0.96$ indicates slight changes in the diagonal terms $A_{11}$ and $A_{22}$. The remaining diagonal terms, $A_{33}$ and $A_{44}$, indicate a more significant shift in the real parts such that the Navier-Stokes forces are smaller in magnitude in comparison with the Euler forces. In general, the real components of the GAF's show a greater sensitivity to viscosity than do the imaginary components. Figure 10 indicates that the same is true for the GAF's at $M_{\infty}=1.141$. However, in contrast to the results for $M_{\infty}=0.96$, Fig. 10 indicates that the diagonal term $A_{22}$ shows a more significant difference in the real components of the viscous and inviscid forces at the experimental flutter reduced frequency of 0.1. A decrease in the magnitude of the real component of $A_{22}$ is associated with an aft shift of the aerodynamic center of the wing. ${ }^{16}$ Therefore the results shown in Fig. 10 indicate that the effect of viscosity is to delay the aft motion of the aerodynamic center. This is consistent with the results shown in the steady-state surface pressure coefficient contours for $M_{\infty}=1.141$. The diagonal terms $A_{11}, A_{33}$, 

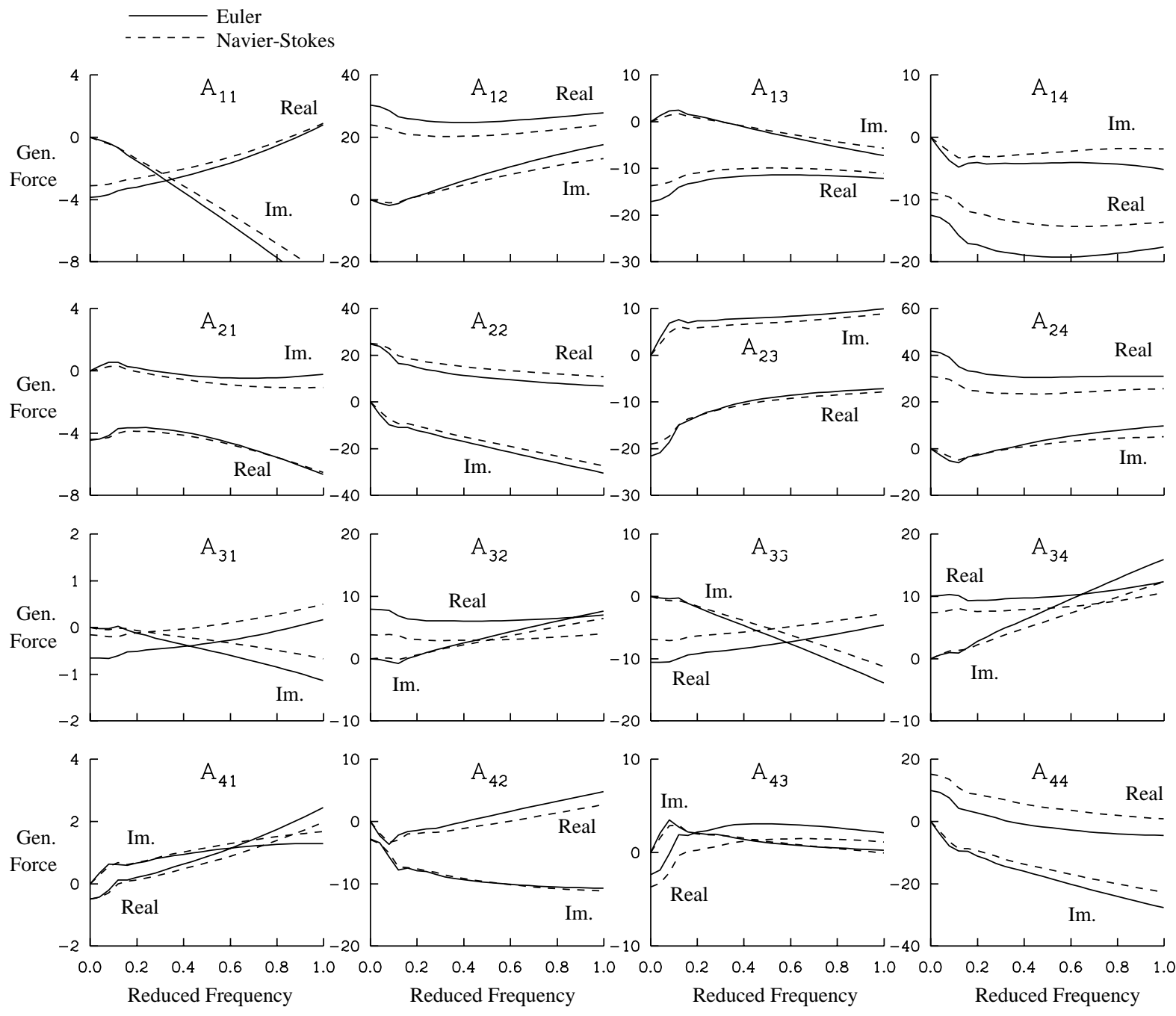

Figure 9 Comparison of generalized aerodynamic forces for the first four structural modes of Wing 445.6 at $M_{\infty}=0.96$ and $\alpha=0^{\circ}$.

and $A_{44}$ in Fig. 10 show similar effects due to viscosity as shown in Fig. 9 for these GAF's.

A comparison of results in Figs. 9 and 10 shows the effects of increasing the free-stream Mach number. The diagonal terms $A_{11}$ and $A_{33}$ exhibit only slight changes with increase in $M_{\infty}$. However, the real component of $A_{22}$ exhibits a decrease from $M_{\infty}=0.96$ to 1.141 which is greater for the GAF's computed from the Euler calculation. As noted, the decrease in the real component of $A_{22}$ indicates an aft shift in the aerodynamic center of the wing. The real component of $A_{44}$ exhibits a change in sign with increasing $M_{\infty}$. The off-diagonal term $A_{21}$ exhibits a decrease in the magnitude of real component with an increase in $M_{\infty}$ which is also an indication of an aft shift in the aerodynamic center. Other off-diagonal terms exhibit changes in sign with increase in $M_{\infty}$.

\section{Aeroelastic Results}

$\underline{\mathrm{V}-\mathrm{g} \text { analysis }}$ Flutter characteristics are determined in a V-g analysis for $M_{\infty}=0.960$ and 1.141 at $\alpha=0^{\circ}$ using the harmonic loads shown in Figs. 9 and 10. Since the wing has a symmetric airfoil section, at zero degrees angle of attack, this configuration has no static aeroelastic deflections. Therefore, static aeroelastic characteristics did not need to be considered.

The linear stability analysis was performed for three modal configurations: modes 1 and 2 ( 2 modes), modes 1 , 2 and 3 (3 modes), and modes 1, 2, 3 and 4 (4 modes). The flutter characteristics determined from these V-g analyses are shown in Figs. 11 and 12. The computed flutter characteristics in terms of flutter speed index $\frac{U_{f}}{b \omega_{\alpha} \sqrt{\mu}}$ and nondimensional flutter frequency ratio $\frac{\omega}{\omega_{\alpha}}$ are shown in Figs. 11 and 12 along with the experimental results. An additional case with structural damping $\left(g_{i}\right)$ was computed for the 4-mode analyses, but since no structural damping was measured for the wind-tunnel model, a estimated value of 0.03 was applied to each mode in order to evaluate the effects of structural damping. The results for $M_{\infty}=0.960$ shown in Fig. 


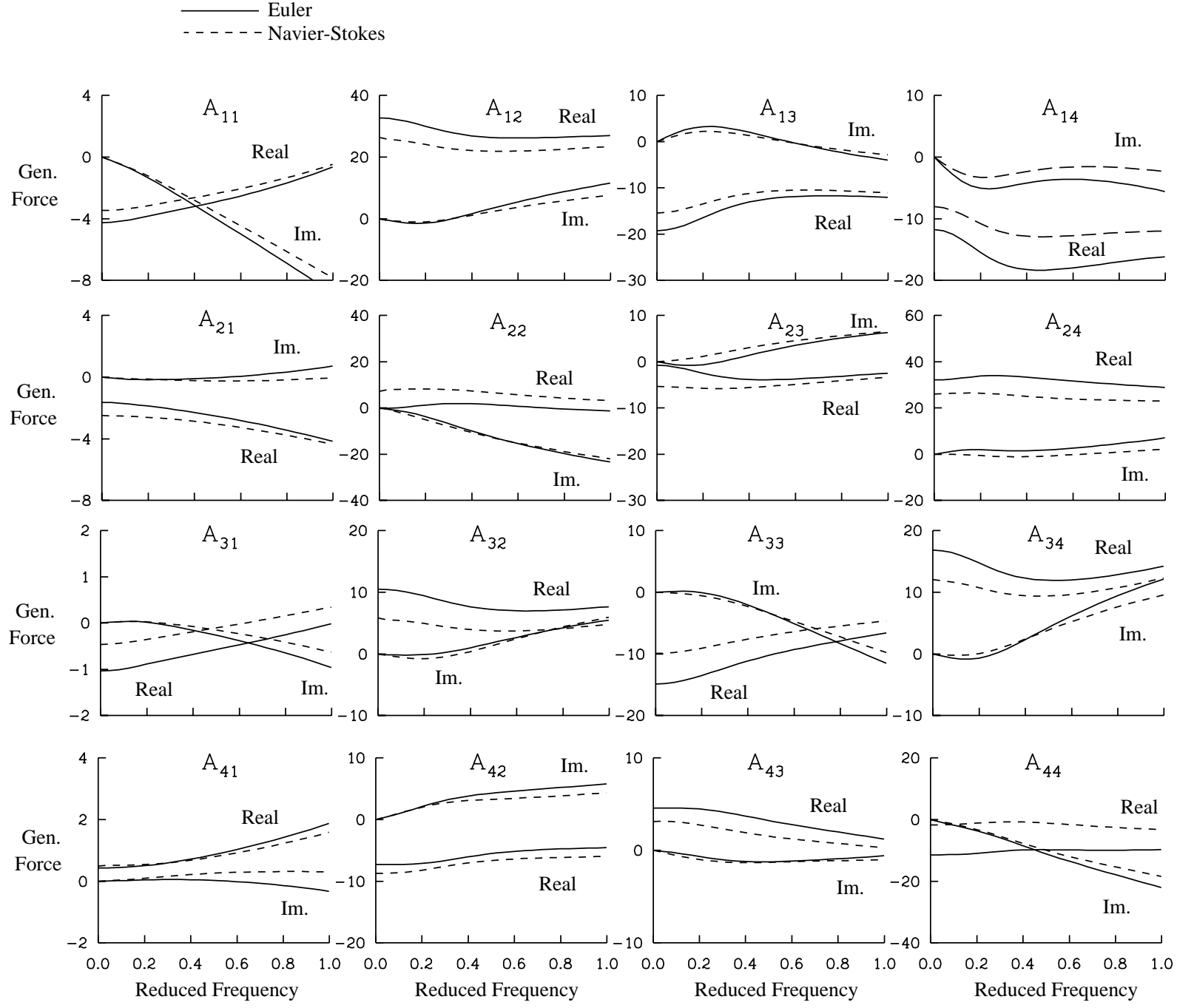

Figure 10 Comparison of generalized aerodynamic forces for the first four structural modes of Wing 445.6 at $M_{\infty}=1.141$ and $\alpha=0^{\circ}$.

11 indicate that modes 3 and 4 do not significantly affect the flutter speed and frequency for either the Euler or the Navier-Stokes results. Figure 11 also indicates that including the effects of viscosity increases the flutter speed index by 9.6 percent for the 4-mode analysis which improves correlation with the experimental data. The addition of structural damping stabilizes the wing which further improves the correlation. The flutter speed index computed from the Navier-Stokes GAF's with structural damping included are within 5 percent of the experimental value of flutter speed index. The 4-mode Euler and Navier-Stokes results (with structural damping) are shown in Fig. 13 along with the experimental boundary and the flutter boundary predicted in Ref. 13. The present 4-mode Euler flutter analysis predicts a lower flutter speed (approximately 7 percent) than that predicted in Ref. 13. This is thought to be due to the increase in spatial resolution of the Euler grid used in the present analyses. The Reynolds numbers at flutter predicted in the V-g analyses with the Navier-Stokes GAF's range from 374,000 per foot to 382,000 per foot, a maximum of 4.5 percent difference from the experimental free-stream Reynolds number $\left(Q / Q_{e x p}=0.86-0.92\right)$.

The results shown in Fig. 12 indicate that modes 3 and 4 have a greater effect on the flutter characteristics for the Euler and Navier-Stokes results at $M_{\infty}=1.141$. Based on the results of the 2-mode analysis, the significant decrease in the real component of $A_{22}$ noted in the Euler pulse analyses from $M_{\infty}=0.96$ to 1.141 provides the stabilizing aerodynamics that produced the rapid rise on the flutter boundary in this Mach number range. Including modes 3 and 4 in the analysis tends to further stabilize the wing. A comparison between the flutter characteristics predicted with the Euler and Navier-Stokes GAF's in Fig. 12 indicates that including the effects of viscosity significantly improves the correlation with the experimental results. In the 4-mode analysis, the flutter speed index predicted with the NavierStokes GAF's is lower than the flutter speed index predicted 


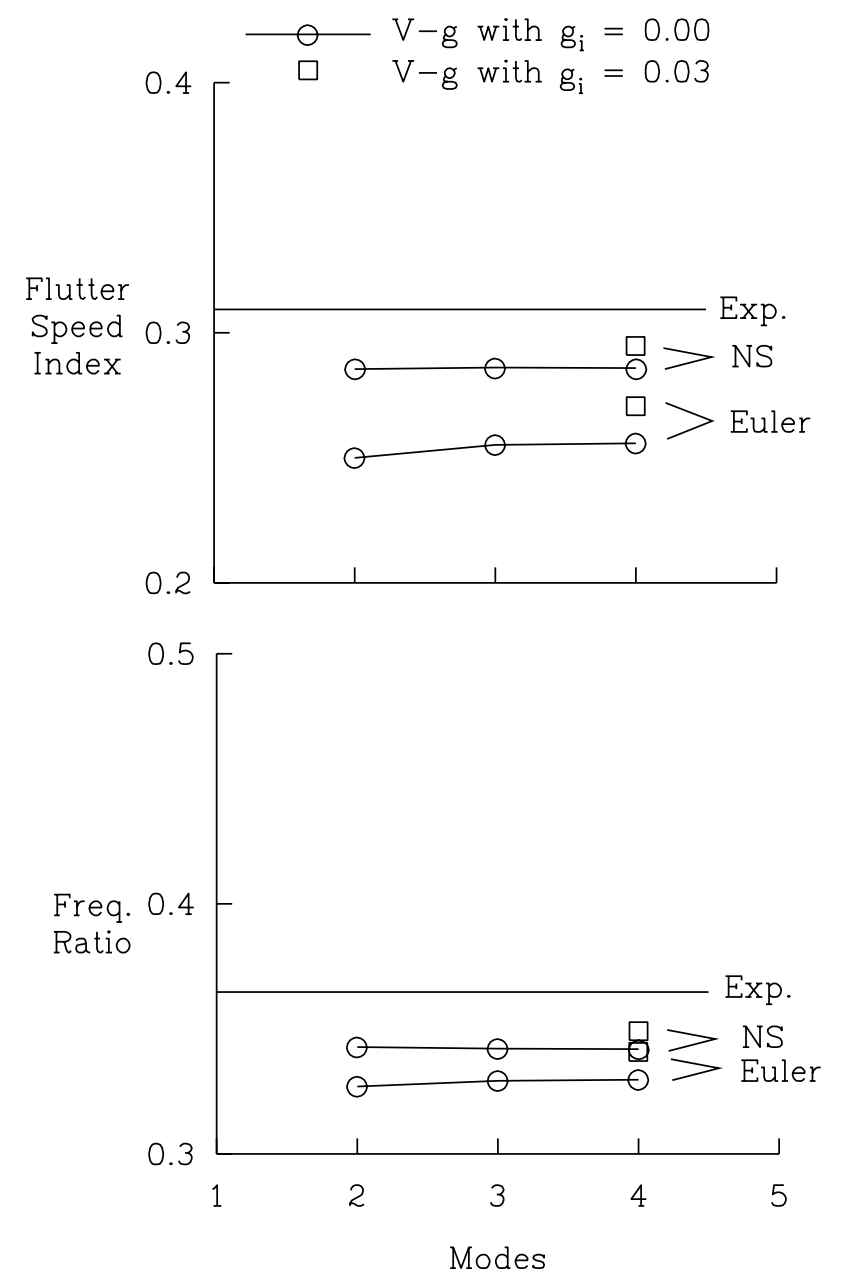

Figure 11 Comparison of computed flutter characteristics for Wing 445.6 at $M_{\infty}=0.96$ and $\alpha=0^{\circ}$.

with the Euler GAF's by 46 percent of the experimental value. Figure 12 also shows that the inclusion of structural damping destabilizes the wing at this Mach number which further improves the correlation with the experiment. (The fact that the addition of structural damping destabilizes the wing is somewhat unusual). However, the flutter speed index computed using the Navier-Stokes GAF's with structural damping is 17.5 percent higher than the experimental value. The 4-mode Euler and Navier-Stokes results (with structural damping) are shown in Fig. 13 along with the experimental boundary and the flutter boundary predicted in Ref. 13. The present Euler flutter analysis predicts flutter at a slightly higher speed (approximately 3 percent) than that predicted in Ref. 13. This is also thought to be due to the increase in spatial resolution of the Euler grid used in the present analyses. The Reynolds numbers at flutter predicted in the V-g analyses with the Navier-Stokes GAF's range from 486,000 per foot to 502,000 per foot, a maximum of 9.6 percent difference from the experimental free-stream Reynolds number $\left(Q / Q_{e x p}=1.28-1.46\right)$.
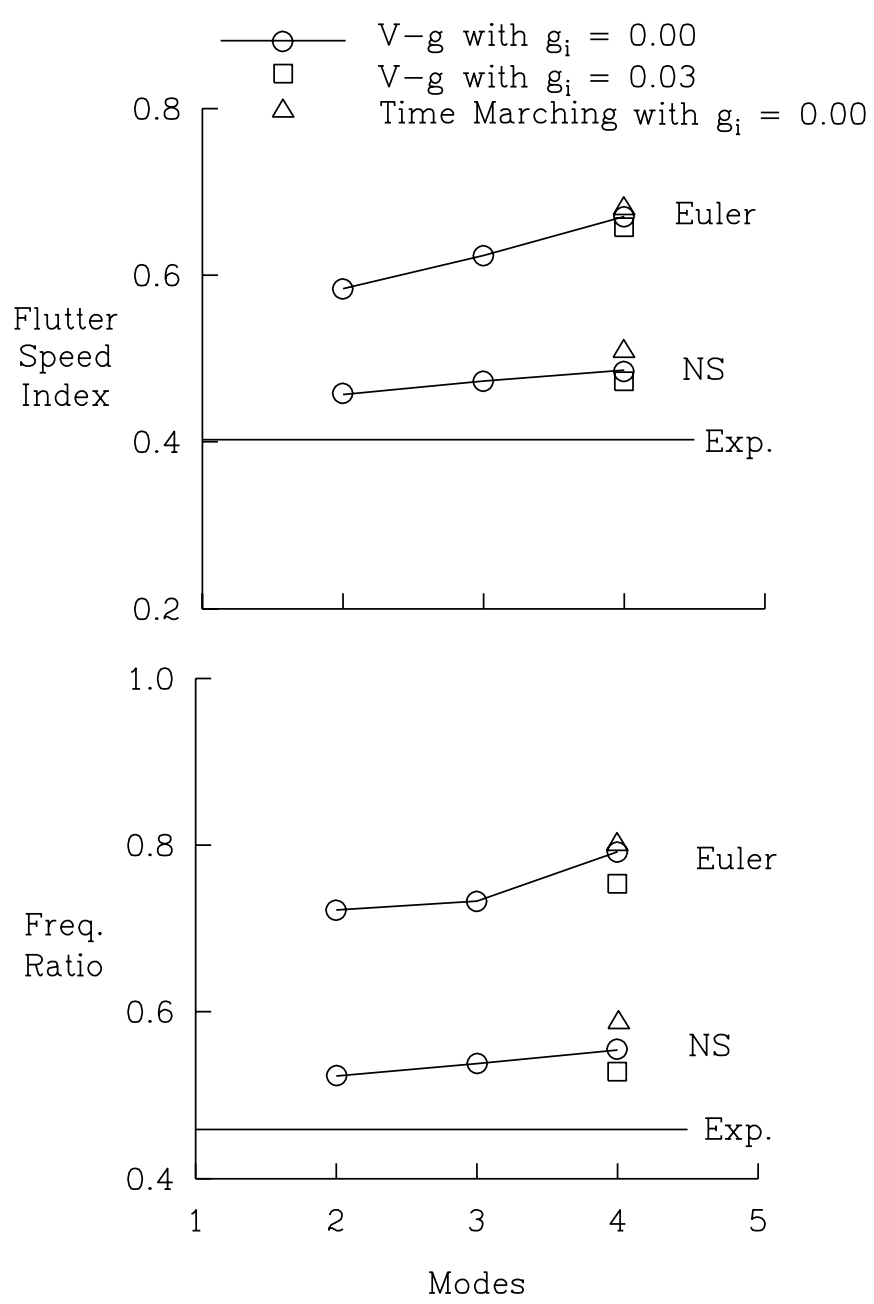

Figure 12 Comparison of computed flutter characteristics for Wing 445.6 at $M_{\infty}=1.141$ and $\alpha=0^{\circ}$.

Time-marching analysis Time-marching calculations are performed at $M_{\infty}=1.141$ using the Euler and NavierStokes equations because more significant discrepancies were noted between the computed and experimental results at this free-stream Mach number than at $M_{\infty}=0.96$. In the time-marching analyses, the first four modes are used to model the structure. As mentioned previously, the viscous time-marching calculations are performed with and without variation in the free-stream Reynolds number at dynamic pressures ranging from $Q / Q_{e x p}=1.4$ to 1.85 (flutter dynamic pressure predicted at $\left.Q / Q_{e x p}=1.61\right)$. The flutter results are, however, independent of this change (less than 1 percent difference). The results of the Euler and NavierStokes time-marching aeroelastic analyses are included with the V-g results shown in Fig. 12. The flutter speed index and flutter frequency from the Euler time-marching analysis compare closely with the results of the Euler V-g (4-mode) analysis. The flutter characteristics predicted by the NavierStokes time-marching analysis are high in comparison with the Navier-Stokes V-g (4-mode) results by approximately 5 percent. An additional Navier-Stokes time-marching calcu- 
lation performed at $M_{\infty}=1.141$ included viscous structural damping added to all four modes. The magnitude of the viscous damping corresponded to the amount of $g_{i}$ added in the $\mathrm{V}-\mathrm{g}$ analysis. The addition of the viscous type of structural damping to the time-marching analysis predicted a drop in the flutter speed index which is the same percentage drop as that predicted from the $\mathrm{V}-\mathrm{g}$ analysis.

Although the additional modeling of the viscous boundary layer did improve the correlation with the experiment for the cases considered in this study, several issues concerning the modeling of this aeroelastic problem still need to be addressed. Since the wind tunnel test was performed under free-transition conditions, information concerning transition over the wing model is unknown, and therefore, it is difficult to evaluate the accuracy of the modeling of the boundary layer. In addition, accurate modeling of the tip geometry (the wind tunnel model has a cut-off tip) is difficult for body-fitted meshes, and the effect of this loss in geometric definition on the tip aerodynamics is unknown. The results of the V-g analysis also indicate that higher modes might be needed in the computational model at the higher $M_{\infty}$. The effects of in-plane deflections are an additional unknown.

\section{Conclusions}

The flutter characteristics of an isolated $45^{\circ}$ swept-back wing, Wing 445.6, were studied using an unsteady Euler and Navier-Stokes algorithm in order to investigate a previously noted discrepancy between Euler flutter characteristics and the experimental data. The algorithm, which is a three-dimensional, implicit, upwind Euler/Navier-Stokes code (CFL3D Version 2.1), was previously modified for the time-marching, aeroelastic analysis of wings using the unsteady Euler equations. These modifications included the incorporation of a deforming mesh algorithm and the addition of the structural equations of motion for their simultaneous time integration with the governing flow equations. In this paper, the aeroelastic method was extended and evaluated for applications using Navier-Stokes aerodynamics. The paper presented a brief description of the aeroelastic method and presented unsteady calculations which verified the method for Navier-Stokes calculations. A linear stability analysis and a time-marching aeroelastic analysis were used to determine the flutter characteristics of the wing. Effects of fluid viscosity, structural damping and number of modes in the structural model were investigated.

In the linear stability analysis, the unsteady generalized aerodynamic forces of the wing were computed using the Euler and Navier-Stokes equations for a range of reduced frequency using the pulse transfer-function method. The flutter characteristics of the wing were determined using these unsteady generalized aerodynamic forces in a traditional V-g flutter analysis at free-stream Mach numbers of 0.96 and 1.141. The results of the V-g analysis indicated that the effect of viscosity on the flutter characteristics was
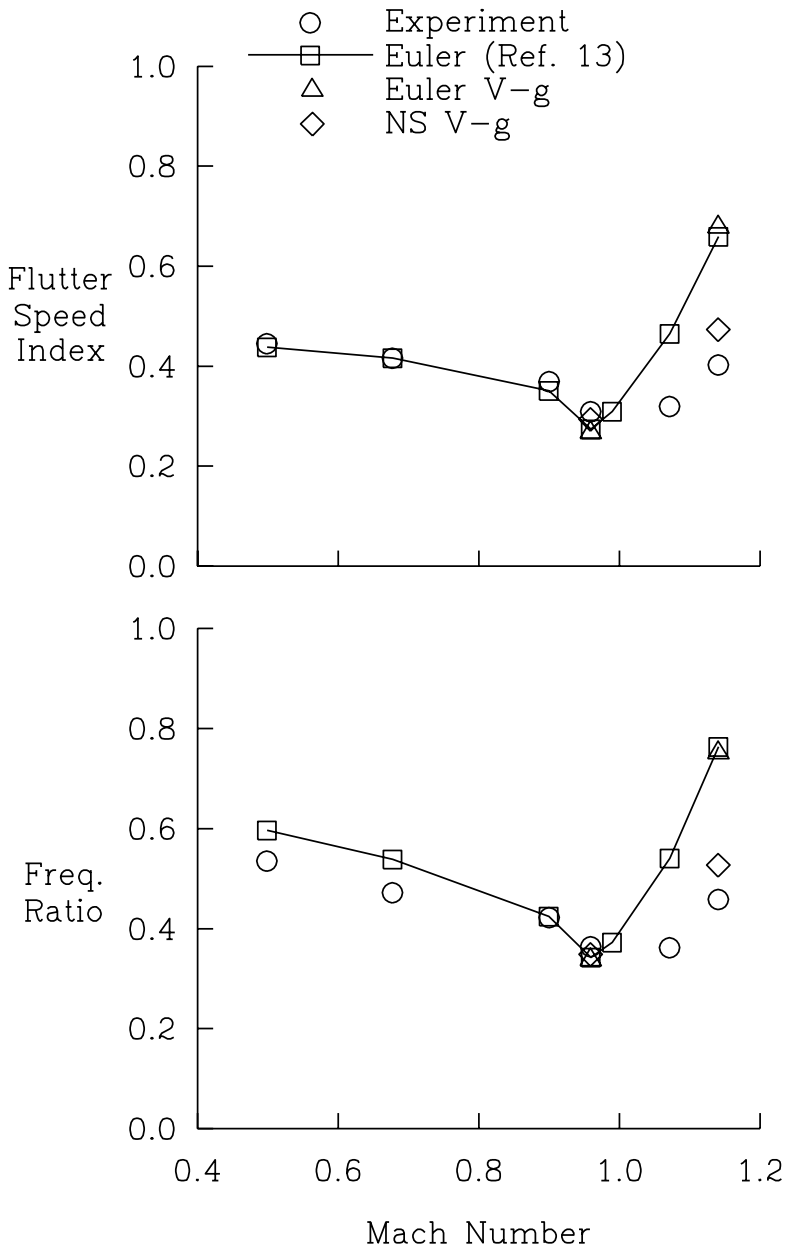

Figure 13 Comparison of Euler and Navier-Stokes $\mathrm{V}-\mathrm{g}$ results with Euler flutter results from Ref. 13 and experimental data for Wing 445.6.

to delay the rise in the flutter boundary on the supersonic side which significantly improved correlation with the experimental boundary. The V-g analysis also indicated that the effect of structural damping was to delay the rise in the boundary as well. Although the flutter characteristics predicted by the Navier-Stokes V-g analysis for the free-stream Mach number of 0.96 compared very well with the experimental data, the results for the free-stream Mach number of 1.141 were still high in comparison with the experimental data. The effect on the flutter characteristics of including higher structural modes in the V-g analysis was less significant at the free-stream Mach number of 0.96 than at the free-stream Mach number of 1.141.

Time-marching aeroelastic calculations were performed at a free-stream Mach number of 1.141 using the Euler and Navier-Stokes equations to compare with the linear Vg flutter analysis method. The Euler results for the timemarching analysis were within 2 percent of the flutter speed predicted by the V-g analysis, and the Navier-Stokes results for the time-marching analysis were within 5 percent of the flutter speed predicted by the V-g analysis. Although the 
linear stability analyisis flutter results did not match the timemarching results, the $\mathrm{V}-\mathrm{g}$ analysis did, however, indicate the significant effect of viscosity on the supersonic flutter boundary for this wing.

\section{Acknowledgments}

The authors would like to thank Brian A. Robinson of McDonnell Aircraft Company, St. Louis, Missouri, for sharing his experiences concerning the aeroelastic version of CFL3D. The authors would also like to thank James L. Thomas, W. Kyle Anderson and Christopher L. Rumsey of the Computational Aerodynamics Branch, NASA Langley Research Center, and Sherrie L. Krist of ViGYAN Inc., Hampton, Virginia, for their many helpful discussions concerning CFL3D. Finally, the authors would like to thank their colleague Robert M. Bennett for sharing his many insights into computational aeroelasticity and flutter.

\section{$\underline{\text { References }}$}

[1] Ballhaus, W. F; and Goorjian, P. M., "Computation of Unsteady Transonic Flows by the Indicial Method," AIAA Journal, vol. 16, no. 2, pp. 117-124, 1978.

[2] Edwards, J. W.; and Malone, J. B., "Current Status of Computational Methods for Unsteady Transonic Unsteady Aerodynamics and Aeroelastic Analysis," Presented at the AGARD Structures and Materials Panel Specialist's Meeting on Transonic Unsteady Aerodynamics and Aeroelasticity, Paper No. 1, October 1991.

[3] Guruswamy, G. P., "Time-Accurate Unsteady Aerodynamic and Aeroelastic Calculations of Wings Using Euler Equations," AIAA Paper No. 88-2281, April 1988.

[4] Guruswamy, G. P., "Numerical Simulation of Vortical Flows on Flexible Wings," AIAA Paper No. 89-0537, January 1989.

[5] Guruswamy, G. P., "Vortical Flow Computations on Swept Flexible Wings Using Navier-Stokes Equations," AIAA Paper No. 89-1183, April 1989.

[6] Schuster, D.; Vadyak, J.; and Atta, E., "Static Aeroelastic Analysis of Fighter Aircraft Using a ThreeDimensional Navier-Stokes Algorithm," AIAA Paper No. 90-0435, January 1990.

[7] Guruswamy, G. P., "Navier-Stokes Computations on Swept-Tapered Wings, Including Flexibility," AIAA Paper No. 90-1152, April 1990.

[8] Obayashi, S.; Guruswamy, G. P.; and Goorjian, P., "Application of a Streamwise Upwind Algorithm for Unsteady Transonic Computations Over Oscillating Wings," AIAA Paper No. 90-3103, August 1990.

[9] Guruswamy, G. P., "Vortical Flow Computations on a Flexible Blended Wing-Body Configuration," AIAA Paper No. 91-1013-CP, April 1991.
[10] Obayashi, S.; and Guruswamy, G. P., "Unsteady ShockVortex Interaction on a Flexible Delta Wing," AIAA Paper No. 91-1109, April 1991.

[11] Robinson, B. A.; Batina, J. T.; and Yang, H. T. Y., "Aeroelastic Analysis of Wings Using the Euler Equations with a Deforming Mesh," Journal of Aircraft, vol. 28, pp. 778-788, November 1991.

[12] Rausch, R. D.; Batina, J. T.; and Yang, H. T. Y., “ThreeDimensional Time-Marching Aeroelastic Analyses Using An Unstructured-Grid Euler Method ," AIAA Paper No. 92-2506, April 1992.

[13] Lee-Rausch, E. M.; and Batina, J. T., "Wing Flutter Boundary Prediction Using Unsteady Euler Method," AIAA Paper No. 93-1422, April 1993.

[14] Edwards, J. W.; Bennett, R. M.; Whitlow, W., Jr; and Seidel, D. A., "Time-Marching Transonic Flutter Solutions Including Angle-of-Attack Effects," AIAA Paper No. 82-3685, May 1982.

[15] Yang, T. Y.; and Batina, J. T., "Transonic TimeResponse Analysis of 3-Degree-of-Freedom Conventional and Supercritical Airfoils," Journal of Aircraft, vol. 20, no. 8, pp. 703-710, 1983.

[16] Mohr R. W.; Batina J. T.; and Yang, H. T. Y., "Mach Number Effects on Transonic Aeroelastic Forces and Flutter Characteristics," Journal of Aircraft, vol. 26, pp. 1038-1046, November 1989.

[17] Rausch, R. D.; Batina, J. T.; and Yang, H. T. Y., "Spatial Adaption Procedures on Unstructured Meshes for Accurate Unsteady Aerodynamic Flow Computation," AIAA Paper No. 91-1106, April 1991.

[18] Cunningham, H. J.; Batina, J. T.; and Bennett, R. M., "Modern Wing Flutter Analysis by Computational Fluid Dynamics Methods," Journal of Aircraft, vol. 25, no. 10, pp. 962-968, 1988.

[19] Bennett, R. M.; and Desmarais, R. N., "Curve Fitting of Aeroelastic Transient Response Data with Exponential Functions," Flutter Testing Techniques NASA SP-415, May 1975.

[20] Desmarais, R. N.; and Bennett, R. M., “User's Guide for a Modular Flutter Analysis Software System (FAST Version 1.0)," NASA TM 100492, May 1978.

[21] Desmarais, R. N.; and Bennett, R. M., "An Automated Prodedure for Computing Flutter Eigenvalues," Journal of Aircraft, vol. 11, no. 2, pp. 75-80, 1974.

[22] Seidel, D. A.; Bennett, R. M.; and Ricketts, R. H., "Some Recent Applications of XTRAN3S," AIAA Paper No. 83-1811, July 1983.

[23] Rausch, R. D.; Batina, J. T.; and Yang, H. T. Y., "Euler Flutter Analysis of Airfoils Using Unstructured Dynamic Meshes," Journal of Aircraft, vol. 27, no. 5, pp. 436-443, 1990.

[24] Anderson, W. K.; Thomas, J. L.; and van Leer, B., "Comparison of Finite Volume Flux Vector Splitting 
for the Euler Equations," AIAA Journal, vol. 24, no. 9, pp. 1453-1460, 1986.

[25] Anderson, W. K.; Thomas, J. L.; and Rumsey, C. L., "Extension and Applications of Flux-Vector Splitting to Unsteady Calculations on Dynamic Meshes," AIAA Paper No. 87-1152, June 1987.

[26] Rumsey, C. L.: and Anderson, W. K., "Parametric Study of Grid Size, Time Step, and Turbulence Modeling on Navier-Stokes Computations Over Airfoils," Paper No. 5, AGARD CP-437, May 1988.

[27] Baldwin, B. ; and Lomax, H., "Thin Layer Approximation and Algebraic Model for Separated Turbulent Flows," AIAA Paper No. 78-257, January 1978.

[28] Johnson, D., "Predictions of Transonic Separated Flow with an Eddy-Viscosity/Reynolds-Shear-Stress Closure Model," AIAA Paper No. 85-1683, 1985.

[29] Batina, J. T., "Unsteady Euler Algorithm With Unstructured Dynamic Mesh for Complex-Aircraft Aeroelastic Analysis," AIAA Paper No. 89-1189, April 1989.

[30] Yates, E. C., Jr., “AGARD Standard Aeroelastic Configuration for Dynamic Response, Candidate Configuration I.-Wing 445.6," NASA TM 100492, August 1987.

[31] Yates, E. C., Jr.; Land, N. S.; and Foughner, J. T., Jr., "Measured and Calculated Subsonic and Transonic Flutter Characteristics of a 45 deg Sweptback Wing Planform in Air and in Freon-12 in the Langley Transonic Dynamics Tunnel," NASA TN D-1616, March 1963. 
